\title{
INEQUALITIES RELATING SECTIONAL CURVATURES OF A SUBMANIFOLD TO THE SIZE OF \\ ITS SECOND FUNDAMENTAL FORM AND APPLICATIONS TO PINCHING THEOREMS FOR SUBMANIFOLDS
}

\author{
RALPH HOWARD AND S. WALTER WEI
}

\begin{abstract}
The Gauss curvature equation is used to prove inequalities relating the sectional curvatures of a submanifold with the corresponding sectional curvature of the ambient manifold and the size of the second fundamental form. These inequalities are then used to show that if a manifold $\bar{M}$ is $\delta$-pinched for some $\delta>\frac{1}{4}$, then any submanifold $M$ of $\bar{M}$ that has small enough second fundamental form is $\delta_{M}$-pinched for some $\delta_{M}>\frac{1}{4}$. It then follows from the sphere theorem that the universal covering manifold of $M$ is a sphere. Some related results are also given.
\end{abstract}

1. Introduction. This note is motivated by questions of the following type: Let $\bar{M}$ be a complete Riemannian manifold and $M$ a compact immersed submanifold of $\bar{M}$; how then is the topology of $M$ affected by placing a sufficiently small upper bound on the size of the second fundamental form of $M$ in $\bar{M}$ ? For example, when $\bar{M}$ is isometric to a standard sphere, Lawson and Simons $[\mathbf{L}-\mathbf{S}]$ show that if the length of the second fundamental form of $M$ is small enough, then $M$ is a homotopy sphere. If $\bar{M}$ is the product of two spheres, then the second author has shown in [Wei] that the submanifolds of $\bar{M}$ with sufficiently small second fundamental are homeomorphic to totally geodesic submanifolds of $\bar{M}$.

Here we will consider the case that $\bar{M}$ is $\delta$-pinched for some $\delta>\frac{1}{4}$. That is, all sectional curvatures of $\bar{M}$ are in the closed interval $\left[\delta K_{0}, K_{0}\right]$ for some constant $K_{0}>0$. In this case the well-known sphere theorem of Berger, Klingenberg, Rauch and Toponogov implies that the universal covering manifold of $\bar{M}$ is homeomorphic to a sphere. If $\bar{M}$ and $M$ are both simply connected and $M$ has codimension one, then Flaherty has given conditions (cf. $§ 3$ below) on the second fundamental form of $M$ which forces $M$ to be a homotopy sphere.

In this note we will extend this to higher codimensions and at the same time weaken the assumptions on the second fundamental form of $M$ and drop the assumption of simple connectivity on $\bar{M}$.

Our method is to use the Gauss curvature equation to prove inequalities relating the sectional curvatures of a submanifold with the corresponding sectional curvatures of the ambient manifold and the size of the second fundamental form of the submanifold. These inequalities then imply that a submanifold of a pinched manifold is also pinched (with a slightly worse pinching constant) provided that its second fundamental form is small enough. The proofs of these inequalities are elementary; they only involve completing the square.

Received by the editors July 27, 1983 and, in revised form, August 15, 1984 .

1980 Mathematics Subject Classification. Primary 53C40; Secondary 53C20.

(C) 1985 American Mathematical Society $0002-9939 / 85 \$ 1.00+\$ .25$ per page 
This note is an expanded version of a pleasant Saturday afternoon conversation between the authors and Professor Bang-Yen Chen whose help we wish to acknowledge. We would also like to thank the referee for his corrections and suggestions on improving the exposition.

2. The inequalities. Let $M$ be an $n$-dimensional $(n \geq 2)$ submanifold isometrically immersed in the Riemannian manifold $\bar{M}$. At each point $x \in M$ the tangent space to $M$ at $x$ will be written as $T M_{x}$ and the normal space to $M$ at $x$ as $T^{\perp} M_{x}$. The second fundamental form $h_{x}$ of $M$ in $\bar{M}$ at $x$ is a symmetric bilinear form $T M_{x} \times T M_{x}$ to $T^{\perp} M_{x}$. If $e_{1}, \ldots, e_{n}$ is any orthonormal basis on $T M_{x}$, then the length of $h_{x}$ is defined by

$$
\left\|h_{x}\right\|^{2}=\sum_{1 \leq i, j \leq n}\left\|h_{x}\left(e_{i}, e_{j}\right)\right\|^{2} .
$$

If $P$ is a plane section of $M$ at $x$, i.e. a two-dimensional subspace of $T M_{x}$, then denote by $\bar{K}(P)$ the sectional curvature of $\bar{M}$ at $P$, by $K(P)$ the sectional curvature of $M$ at $P$ and by $\left.h\right|_{P}$ the symmetric bilinear from $P \times P$ to $T^{\perp} M_{x}$ obtained by restricting $h_{x}$ to $P \times P$. Let $e_{1}, e_{2}$ be any orthonormal basis of $P$. Then the Gauss curvature equation can be written as

$$
K(P)=\bar{K}(P)+\left\langle h\left(e_{1}, e_{1}\right), h\left(e_{2}, e_{2}\right)\right\rangle-\left\|h\left(e_{1}, e_{2}\right)\right\|^{2}
$$

and the length of $\left.h\right|_{P}$ is

$$
\begin{aligned}
\left\|\left.h\right|_{P}\right\|^{2} & =\sum_{1 \leq i, j \leq 2}\left\|h\left(e_{i}, e_{j}\right)\right\|^{2} \\
& =\left\|h\left(e_{1}, e_{1}\right)\right\|^{2}+2\left\|h\left(e_{1}, e_{2}\right)\right\|^{2}+\left\|h\left(e_{2}, e_{2}\right)\right\|^{2} .
\end{aligned}
$$

Clearly $\left\|\left.h\right|_{P}\right\|^{2} \leq\left\|h_{x}\right\|^{2}$. Our estimates are

Proposition 1. If $P$ is a plane section of $M$, then

$$
\begin{aligned}
\bar{K}(P)-\frac{1}{2}\|h\|^{2} & \leq \bar{K}(P)-\frac{1}{2}\left\|\left.h\right|_{P}\right\|^{2} \leq K(P) \\
& \leq \bar{K}(P)+\frac{1}{2}\left\|\left.h\right|_{P}\right\|^{2} \leq \bar{K}(P)+\frac{1}{2}\|h\|^{2} .
\end{aligned}
$$

Proposition 2. If $M$ is a minimal surface in $\bar{M}$, then

$$
\bar{K}(P)-\frac{1}{2}\|h\|^{2}=K(P) \leq \bar{K}(P) .
$$

Proposition 3. If $M$ is a totally umbilic surface in $\bar{M}$, then

$$
\bar{K}(P) \leq K(P)=\bar{K}(P)+\frac{1}{2}\|h\|^{2} .
$$

PROPOSITION 4. If $\bar{M}$ is a Kaehler manifold and $M$ is a Kaehler submanifold of $\bar{M}$, then for every holomorphic plane section $P$ of $M$

$$
\bar{K}(P)-\frac{1}{2}\|h\|^{2} \leq \bar{K}(P)-\frac{1}{2}\left\|\left.h\right|_{P}\right\|^{2}=K(P) \leq \bar{K}(P) .
$$

REMARKS. Propositions 2 and 3 show that the inequalities in Proposition 1 are sharp in the case that $M$ is two-dimensional. By considering cylinders over minimal surfaces or umbilic surfaces in Euclidean space it is possible to show that the inequalities in Proposition 1 are sharp in all dimensions. Proposition 4 is a restatement of Proposition 9.2 in Volume 2 of $[\mathbf{K}-\mathbf{N}]$. It is included here because of its relation to the other results. 
PROOF. Let $e_{1}, e_{2}$ be an orthonormal basis of $P$. Let $X=h\left(e_{1}, e_{1}\right), Y=$ $h\left(e_{1}, e_{2}\right)$ and $Z=h\left(e_{2}, e_{2}\right)$. Because of equations (2) and (3), to prove Proposition 1 it is enough to show that

$$
-\left(\|X\|^{2}+2\|Y\|^{2}+\|Z\|^{2}\right) \leq 2\left(\langle X, Z\rangle-\|Y\|^{2}\right) \leq\|X\|^{2}+2\|Y\|^{2}+\|Z\|^{2} .
$$

This follows at once from the identities

$$
\begin{gathered}
\|x\|^{2}+2\|Y\|^{2}+\|Z\|^{2}-2\left(\langle X, Z\rangle-\|Y\|^{2}\right)=\|X-Z\|^{2}+4\|Y\|^{2} \geq 0 \\
2\left(\langle X, Z\rangle-\|Y\|^{2}\right)+\|X\|^{2}+2\|Y\|^{2}+\|Z\|^{2}=\|X+Z\|^{2} \geq 0
\end{gathered}
$$

If $M$ is a minimal surface and $x \in M$, then let $e_{1}, e_{2}$ be an orthonormal basis of $T M_{x}$. Because $M$ is minimal the mean curvature vector of $M$ is zero so $0=$ $h\left(e_{1}, e_{1}\right)+h\left(e_{2}, e_{2}\right)=X+Z(X, Y, Z$ as above). Using $Z=-X$ in (2) yields $K(P)=\bar{K}(P)-\|X\|^{2}-\|Y\|^{2}$ and in (1) it yields $\|h\|^{2}=2\|X\|^{2}+2\|Y\|^{2}$. These two equations imply Proposition 2.

If $M$ is a totally umbilic surface, then by definition $Y=h\left(e_{1}, e_{2}\right)=0$ and $X=h\left(e_{1}, e_{1}\right)=h\left(e_{2}, e_{2}\right)=Z$. Thus $K(P)=\bar{K}(P)+\|X\|^{2}$ and $\|h\|^{2}=2\|X\|^{2}$. This proves Proposition 3.

3. Submanifolds of pinched manifolds. If $M$ is a Riemannian manifold and $0<\delta \leq 1$, then $M$ is said to be $\delta$-pinched if and only if there is a positive constant $K_{0}$ such that $\delta K_{0} \leq K(P) \leq K_{0}$ for all plane sections $P$ of $M$. It is clear that the above results can be used to relate pinching (or holomorphic pinching) of a manifold to pinching (or holomorphic pinching) of its submanifolds. For example, Proposition 1 easily implies

Proposition 5. Let $\bar{M}$ be a Riemannian manifold with $\delta \leq \bar{K}(P) \leq 1$ for all plane sections of $P$ of $\bar{M}$ and let $M$ be a submanifold of $\bar{M}$ so that $\left\|\left.h\right|_{P}\right\|^{2} \leq B^{2}$ for all plane sections $P$ of $M$. Then all the sectional curvatures of $M$ are in the interval $\left[\delta-\frac{1}{2} B^{2}, \delta+\frac{1}{2} B^{2}\right]$. Thus if $B^{2}<2 \delta$, then $M$ is $\delta_{M}$-pinched with

$$
\delta_{M}=\frac{\delta-B^{2} / 2}{1+B^{2} / 2}=\frac{2 \delta-B^{2}}{2+B^{2}} .
$$

COROLlARY. If $\delta>\frac{1}{4}$ and $M$ is complete with $\left\|\left.h\right|_{P}\right\|^{2} \leq(8 \delta-2) / 5$ for all plane sections $P$ of $M$, then $M$ is $\delta_{M}$-pinched for some $\delta_{M}>\frac{1}{4}$ and thus its universal covering manifold is homeomorphic to a sphere.

We now give a statement and an elementary proof of the theorem of Flaherty mentioned above.

THEOREM $[\mathbf{F}]$. Let $\bar{M}$ be a complete, simply connected, Riemannian manifold of dimension at least three that has all its sectional curvatures in the interval $[\delta, 1]$ with $\delta>\frac{1}{4}$ (this implies $\bar{M}$ is homeomorphic to a sphere). Let $M$ be a simply connected hypersurface of $\bar{M}$ such that the second fundamental forms of $M$ with respect to one of the two outward unit normals have their eigenvalues in $[0, B]$, where $B<\cot (\pi /(4 \sqrt{\delta}))$. Then $M$ is a homotopy sphere.

To prove this theorem we first note that if all of the eigenvalues of the second fundamental form of a hypersurface $M$ are in the interval $[0, B]$ for one of the two 
choices of the outward normal, then for all plane sections $P$ of $M$,
(A) $K(P) \geq \bar{K}(P)$,
(B) $\left\|\left.h\right|_{P}\right\|^{2} \leq 2 B^{2}$.

(The first follows from the Gauss equation and the assumption that the eigenvalues are $\geq 0$. For the second use that eigenvalues of $\left.h\right|_{P}$ are also in the interval $[0, B]$ and so $\left\|\left.h\right|_{P}\right\|^{2}=\lambda_{1}^{2}+\lambda_{2}^{2} \leq 2 B^{2}$.) The conditions (A) and (B) make sense for submanifolds of any codimension.

Proposition 1 now implies

PROPOSITION 6. Let $\bar{M}$ be a Riemannian manifold with all its sectional curvatures in the interval $[\delta, 1]$ with $\delta>0$. Let $M$ be a complete submanifold of $\bar{M}$ that satisfies the conditions (A) and (B). Then the sectional curvatures of $M$ are in the interval $\left[\delta, 1+B^{2}\right]$ and thus $M$ is $\delta_{M}$-pinched with $\delta_{M}=\delta /\left(1+B^{2}\right)$.

COROLlaRY. If $\delta>\frac{1}{4}$ and $B^{2}<4 \delta-1$ in the last proposition, then $M$ is $\delta_{M}$-pinched for some $\delta_{M}>\frac{1}{4}$. Therefore the universal covering manifold of $M$ is a sphere.

To show that this corollary implies Flaherty's theorem, it is enough to show that $\frac{1}{4}<\delta \leq 1$ implies $\cot ^{2}(\pi /(4 \sqrt{\delta}))<4 \delta-1$. Since $0<\cot (\pi /(4 \sqrt{\delta})) \leq 1$ for $\delta$ in the given interval, the required inequality is implied by $\cot (\pi / 4 \sqrt{\delta})<4 \delta-1$. Letting $x=1 \sqrt{\delta}$ we want $f(x)=4 x^{-2}-\cot (\pi x / 4)-1>0$ when $1 \leq x<2$. It is enough to show $f$ has no zero on $[1,2)$. At a zero of $f$, we have $4 x^{-2}-1=\cot (\pi x / 4) \leq 1$. This inequality implies $x \geq \sqrt{2}$. Thus we only need to show $f(x) \neq 0$ on $[\sqrt{2}, 2)$. On this interval

$$
\begin{aligned}
f^{\prime}(x) & =-\frac{8}{x^{3}}+\frac{\pi}{4} \csc ^{2}\left(\frac{\pi}{4} x\right) \leq-\left.\frac{8}{x^{3}}\right|_{x=2}+\left.\frac{\pi}{4} \csc ^{2}\left(\frac{\pi}{4} x\right)\right|_{x=\sqrt{2}} \\
& =-1.0+.978262725<0 .
\end{aligned}
$$

Therefore $f$ is decreasing on $[\sqrt{2}, 2)$ and $f(2)=0$. Consequently, $f(x)>0$ on $[1,2)$ as claimed.

\section{REFERENCES}

[F] F. J. Flaherty, Spherical submanifolds of pinched manifolds, Amer. J. Math. 89 (1967), 1109-1114.

[K-N] S. Kobayashi and K. Nomizu, Foundations of differential geometry. I, II, Wiley Interscience, New York, 1967 and 1969.

[L-S] H. B. Lawson and J. Simons, On the stable currents and their applications to global problems in real and complex geometry, Ann. of Math. 98 (1973), 427-450.

[Wei] S. W. Wei, On topological vanishing theorems and the stability of Yang-Mills fields, Indiana Univ. Math. J. 33 (1984), 511-529.

Department of mathematics, University of South Carolina, Columbia, SOUTH CAROLINA 29208

Department of Mathematics, University of California, los Angeles, CaliFORNIA 90024 